\title{
Unsteady combustion mode with a super- high frequency induced by a curved shock
}

Cite as: Phys. Fluids 32, 116101 (2020); https://doi.org/10.1063/5.0026746

Submitted: 26 August 2020 . Accepted: 12 October 2020 . Published Online: 02 November 2020

Honghui Teng (滕宏辉), (D Shuai Liu (刘帅), and (D) Zijian Zhang (张子健)
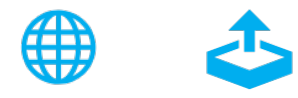

View Online

Export Citation

\section{ARTICLES YOU MAY BE INTERESTED IN}

Shock-wave thickness influence to the light diffraction on a plane shock wave

Physics of Fluids 32, 116103 (2020); https://doi.org/10.1063/5.0029612

Two-stage growth mode for lift-off mechanism in oblique shock-wave/jet interaction

Physics of Fluids 32, 116105 (2020); https://doi.org/10.1063/5.0022449

Numerical study on reflection of an oblique detonation wave on an outward turning wall

Physics of Fluids 32, 046101 (2020); https://doi.org/10.1063/5.0001845

\section{Physics of Fluids MALLERY OF GOVERS}




\title{
Unsteady combustion mode with a super-high frequency induced by a curved shock
}

\author{
Cite as: Phys. Fluids 32, 116101 (2020); doi: 10.1063/5.0026746 \\ Submitted: 26 August 2020 - Accepted: 12 October 2020 • \\ Published Online: 2 November 2020
}

\author{
Honghui Teng (滕宏辉), ' (D) Shuai Liu (刘帅), ' (D) and Zijian Zhang (张子健) ${ }^{2,3, a)}$ (D)

\begin{abstract}
AFFILIATIONS
${ }^{1}$ School of Aerospace Engineering, Beijing Institute of Technology, Beijing 100081, China

${ }^{2}$ School of Engineering Science, University of Chinese Academy of Sciences, Beijing 100049, China

${ }^{3}$ Institute of Mechanics, Chinese Academy of Sciences, Beijing 100190, China
\end{abstract}

a) Author to whom correspondence should be addressed: zhangzijian@imech.ac.ch

\begin{abstract}
Previous studies of a high-speed blunt projectile in a combustible mixture found two oscillating unsteady combustion modes induced by the curved shock, referred to as high- and low-frequency modes. A new unsteady combustion mode is observed in the present study. The frequency reaches approximately twice the high frequency and is referred to as the super-high frequency to maintain consistency with the terminology used in previous works. The super-high-frequency mode appears in cases of a small sphere diameter, and with a proper diameter, an intermediate mode arises with the co-existence of both high and the super-high frequencies. An analysis of pressure and temperature gradients along the stagnation streamline attributes the oscillation of combustion to the interaction of compression and entropy waves between the shock and flame front. If the compression/entropy waves affect the flame front of the next cycle, the high-frequency mode arises; this is consistent with the results of previous works. However, weakened compression/entropy waves in cases of a small sphere diameter only affect the flame front of every other cycle, leading to the super-high-frequency mode.
\end{abstract}

Published under license by AIP Publishing. https://doi.org/10.1063/5.0026746

\section{INTRODUCTION}

Shock-induced combustion occurs widely in natural phenomena and human activities, such as cosmic supernova explosions and catastrophic industrial explosions. ${ }^{1,2}$ In recent years, research on airbreathing engines for use in hypersonic aircrafts, involving different types of shock-induced combustion, has become a frontier in the fields of aeronautics and astronautics. As an example, supersonic combustion occurs behind a train of oblique shocks in scramjets. Meanwhile, a detonation-based engine involves a tight coupling of shock and combustion, and fundamental research of such a coupling will provide foundations for wave control and combustion organization.

There are three regimes of shock and combustion coupling. Weak coupling usually features a weak shock, behind which the temperature rises but without self-ignition, while strong coupling corresponds to a strong shock coupled with instantaneous heat release, resulting in a detonation wave. Between the weak and strong coupling regimes, there might be modest coupling featuring unsteady combustion. ${ }^{9}$ A series of comprehensive studies ${ }^{10-15}$ was conducted in the 1990s, exploring the mechanism of oscillating combustion. It was found that the interaction of the flame front and shock through sonic waves dominates unsteady combustion, and given a fixed inflow Mach number, there may be either a high or low oscillation frequency depending on the spherical diameter. However, the oscillation phenomena have not been fully understood, and only some qualitative rules, such as large diameter corresponding to low frequency, have been developed before. To promote the research on this type of unsteady combustion, more accurate frequency features should be simulated and analyzed, which provides the foundation for the future rigorous quantitative models.

This study simulates the shock-induced unsteady combustion, focusing on the effect of the spherical diameter on the mode of oscillating combustion. Benefiting from high-resolution simulations, we observe a new unsteady combustion mode besides the traditional high-frequency (HF) and low-frequency (LF) oscillations, which has not been reported before. Analysis based on compression and entropy waves reveals a physical interaction along the stagnation 
streamline, improving our understanding of modest shock-induced combustion.

\section{NUMERICAL METHODS}

According to the numerical comparison between threedimensional and two-dimensional axisymmetric flow fields of shock-induced combustion over a sphere conducted by Uda and Matsuo, ${ }^{16}$ the axisymmetric assumption is thought as a reasonable simplification to reproduce the key unsteady features of shockinduced combustion phenomenon over a blunt body. Therefore, the axisymmetric assumption is again employed in this study, and the unsteady flow around the sphere is governed by the two-dimensional unsteady axisymmetric multi-species reactive Euler equation, written as

$$
\frac{\partial \mathbf{U}}{\partial t}+\frac{\partial \mathbf{F}}{\partial x}+\frac{\partial \mathbf{G}}{\partial y}+\mathbf{H}=\mathbf{S}
$$

where $\mathbf{U}, \mathbf{F}, \mathbf{G}, \mathbf{H}$, and $\mathbf{S}$ are, respectively, the vectors of conservative variables, convective fluxes in $x$ - and $y$-directions, inviscid axisymmetric source terms, and chemical source terms. These are expressed as

$$
\begin{gathered}
\mathbf{U}=\left[\begin{array}{c}
\rho \\
\rho u \\
\rho v \\
\rho e \\
\rho Y_{1} \\
\vdots \\
\rho Y_{n s-1}
\end{array}\right], \quad \mathbf{F}=\left[\begin{array}{c}
\rho u \\
\rho u^{2}+p \\
\rho u v \\
(\rho e+p) u \\
\rho u Y_{1} \\
\vdots \\
\rho u Y_{n s-1}
\end{array}\right], \quad \mathbf{G}=\left[\begin{array}{c}
\rho v \\
\rho u v \\
\rho v^{2}+p \\
(\rho e+p) v \\
\rho v Y_{1} \\
\vdots \\
\rho v Y_{n s-1}
\end{array}\right], \\
\mathbf{H}=\frac{1}{y}\left[\begin{array}{c}
\rho v \\
\rho u v \\
\rho v^{2} \\
\rho e+p) v \\
\rho v Y_{1} \\
\vdots \\
\rho v Y_{n s-1}
\end{array}\right], \quad S=\left[\begin{array}{c}
0 \\
0 \\
0 \\
0 \\
\dot{w}_{1} \\
\vdots \\
\dot{w}_{n s-1}
\end{array}\right] .
\end{gathered}
$$

Here, $\rho, u, v$, and $p$ are, respectively, the gas density, velocities in $x$ and $y$-directions, and pressure. $Y_{i}(i=1, \ldots, n s)$ is the mass fraction of the $i$ th species, and $n s$ is the total number of species in the gas mixture. The total specific energy of the gas mixture, $e$, is expressed as

$$
e=h-\frac{p}{\rho}+\frac{1}{2}\left(u^{2}+v^{2}\right)
$$

where $h$ is the specific enthalpy of the gas mixture and can be calculated as

$$
h=\sum_{i=1}^{n s} Y_{i} h_{i},
$$

with $h_{i}$, the specific enthalpy of each species, being obtained from fourth-order temperature polynomial fitting conducted by NASA. ${ }^{17}$
Assuming each species to be a perfect gas, the equation of state of the gas mixture is

$$
p=\sum_{i=1}^{n s} p Y_{i} \frac{R_{0}}{W_{i}} T,
$$

where $T$ is the temperature of the gas mixture, $W_{i}$ is the molar mass of the $i$ th species, and $R_{0}=8.314 \mathrm{~J} /(\mathrm{mol} \mathrm{K})$ is the universal gas constant. In Eq. (2), $\dot{w}_{i}$ is the species mass production rate, and it is modeled adopting the Jachimowski hydrogen combustion mechanism, ${ }^{18}$ involving 19 reversible elementary reactions among nine species [i.e., $n s=9$ in Eq. (2)]; these species are $\mathrm{H}_{2}, \mathrm{H}, \mathrm{O}_{2}, \mathrm{O}, \mathrm{OH}$, $\mathrm{HO}_{2}, \mathrm{H}_{2} \mathrm{O}_{2}, \mathrm{H}_{2} \mathrm{O}$, and $\mathrm{N}_{2}$. This detailed chemical mechanism had been successfully applied in previous shock-induced combustion, ${ }^{15}$ detonation, ${ }^{19,20}$ and scramjet ${ }^{21}$ simulations.

In this study, the above equations are numerically solved adopting the quadrilateral-grid-based finite-volume method. ${ }^{22}$ The numerical flux passing through each cell edge is evaluated using a second-order total-variation diminishing (TVD) scheme based on the Harten-Lax-van Leer contact (HLLC) approximate Riemann solver. ${ }^{23}$ Moreover, a minmod limiter is used to suppress spurious oscillations near discontinuities, while high-order accuracy is retained away from strong discontinuities. The time integration of the above equations is explicitly implemented adopting the fourth-order Runge-Kutta method. In overcoming stiffness problems resulting from the mismatch of time scales between the chemical reaction and flow, an operator-splitting method ${ }^{24}$ is employed to treat the chemical source terms.

This study is based on the classical shock-induced combustion experiment of Lehr $^{9}$ conducted in 1972, where a high-speed projectile that had a hemispherical head body with a diameter of $D=15 \mathrm{~mm}$ was shot with a Mach number of $M a=4.18$ into the stoichiometric $\mathrm{H}_{2}$-air mixture with a pressure of $p=0.421$ atm and a temperature of $T=292 \mathrm{~K}$. A bow-curved shock forms upstream of the projectile, and unsteady combustion of the $\mathrm{H}_{2}$-air mixture induced by the bow-curved shock occurs between the shock and projectile surface. Employing Galilean transformation, the above problem can be equivalent to that a stoichiometric $\mathrm{H}_{2}$-air mixture, having the same Mach number (4.18), pressure (0.421 atm), and temperature $(292 \mathrm{~K})$, flows around a sphere with a diameter $D$, as schematically shown in Fig. 1. In order to investigate the scale effect of this unsteady shock-induced combustion problem, the sphere diameter $D$ is not fixed as Lehr's experiment ${ }^{9}$ but ranges from $8 \mathrm{~mm}$ to $30 \mathrm{~mm}$ in this paper.

Considering the axisymmetric geometry of this problem, the computational domain is schematically shown by the dashed lines in Fig. 1. Since the stagnation region near the symmetry axis is the most important region of unsteady shock-induced combustion, structured quadrilateral-grids are used and clustered to the symmetry axis. Moreover, grids are uniformly distributed on the symmetry axis and are clustered toward the sphere surface away from the symmetry axis. The first grid height near the sphere surface is kept the same as that on the symmetry axis. With the present grid topology, the grid size on the symmetry axis (and near the sphere surface) is the minimum grid size throughout the computational domain and is referred to as the characteristic grid size. Note that the unsteady feature of the shock-induced combustion phenomenon is relative to the waves propagating between the shock and flame 


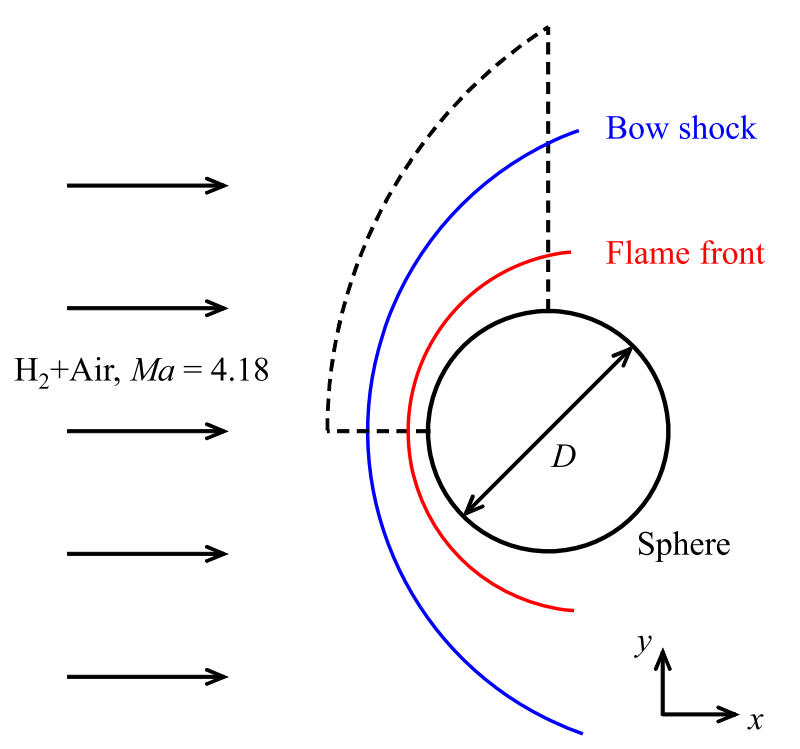

FIG. 1. Schematic of unsteady shock-induced combustion and the computational domain.

front in the stagnation region, whose distance is determined by the length of combustion induction zone along the symmetry axis. ${ }^{10-15}$ In this study, the length of the combustion induction zone varies from about $0.7 \mathrm{~mm}$ to $1.1 \mathrm{~mm}$, depending on the sphere diameter. Therefore, a characteristic grid size of $4 \mu \mathrm{m}$, implying that there are at least about 175 grid points within the combustion induction zone, is adopted in this study, which would be validated by careful grid resolution tests later in Sec. III. In simulations, there is inflow through the upper-left boundary of the computational domain, while there is supersonic outflow through the right boundary. Additionally, the lower boundary, namely, the stagnation line, is set as axisymmetric, while the lower-right boundary is set as an inviscid wall. (a)

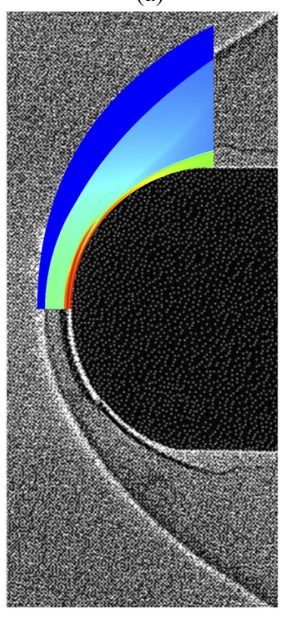

(b)

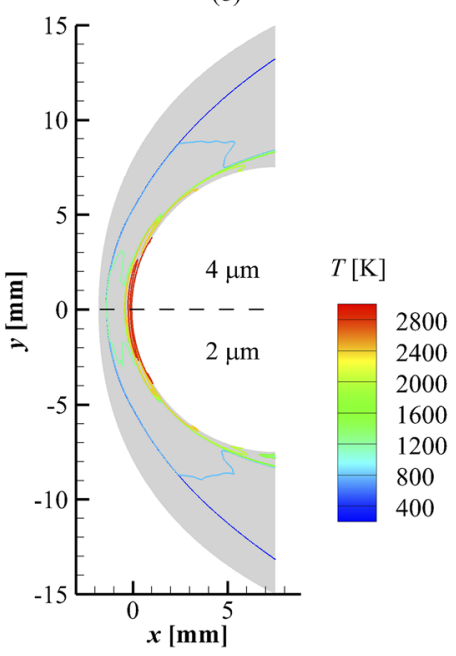

FIG. 2. Comparison of numerical and experimental ${ }^{9}$ flow fields (a) and numerical results obtained for different grid resolutions (b) in the case that $D=15 \mathrm{~mm}$.

\section{RESULTS AND DISCUSSION}

The benchmark case that $D=15 \mathrm{~mm}$ is simulated first, and the results are shown in Fig. 2(a) via temperature contours. It can be clearly seen that a bow-curved shock forms upstream of the sphere, and an intensive heat release region where the temperature rises obviously adjacent to the sphere wall is induced by the bow-curved shock. Figure 3 shows the density and Mach number contours of the simulated flow field around the sphere. A subsonic region with high density forms behind the bow-curved shock near the symmetry axis (also known as the stagnation streamline). Across the flame front, the density decreases abruptly, while the Mach number only decreases slightly. Later in this section, we will demonstrate that

(a)

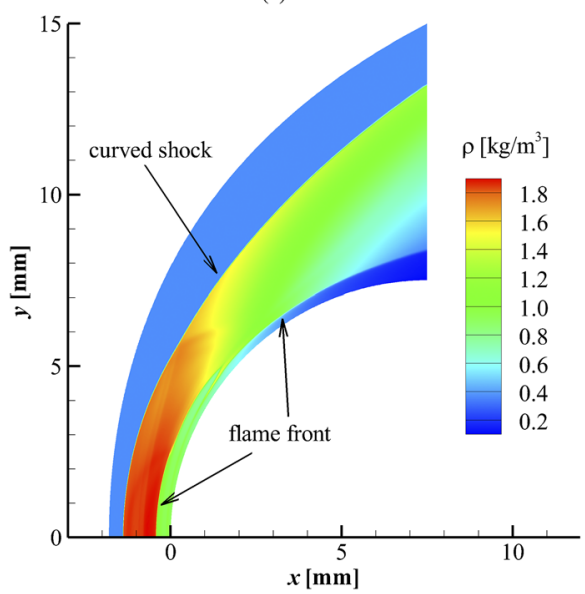

(b)

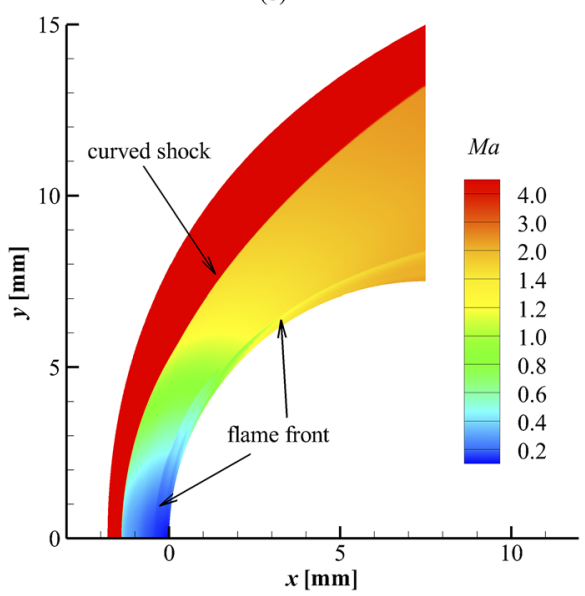

FIG. 3. Contours of density (a) and Mach number (b) in the case that $D=15 \mathrm{~mm}$. 
the unstable flame front would generate various waves propagating in the subsonic region, which results in the unsteady oscillations of the bow-curved shock and flame front. By comparing to the shadowgraph in Lehr's experiment ${ }^{9}$ [also shown in Fig. 2(a)], it can be revealed that the shapes and locations of the simulated bowcurved shock and flame front are nearly identical to those observed in experiment, implying that the above numerical methods are capable of capturing the key flow structures of the present shock-induced combustion problem.

To exclude the influence of grid resolution on the numerical results, grid resolution tests are performed with three characteristic grid sizes, namely, $8 \mu \mathrm{m}, 4 \mu \mathrm{m}$, and $2 \mu \mathrm{m}$. The comparison of the simulated temperature isolines of the flow fields using characteristic grid sizes of $4 \mu \mathrm{m}$ and $2 \mu \mathrm{m}$ is shown in Fig. 2(b). It can be clearly seen that difference between these two flow fields is hard to be distinguished. By recording the time evolution of shock location on the stagnation streamline and processing by adopting the fast Fourier transformation (FFT) technique, the frequencies of the oscillating movement of shock, simulated with the characteristic grid sizes of $8 \mu \mathrm{m}, 4 \mu \mathrm{m}$, and $2 \mu \mathrm{m}$, can be obtained and summarized in Fig. 4 . For comparison, the experimental observation of the oscillation dominant frequency (i.e., $148 \mathrm{kHz}$ ) ${ }^{9}$ is summarized in Fig. 4 as well. It can be revealed that when a characteristic grid size of $8 \mu \mathrm{m}$ is used, the difference in dominant frequency between simulation and experiment is about $9.7 \%$. When the characteristic grid size decreases to $4 \mu \mathrm{m}$ or $2 \mu \mathrm{m}$, the difference accordingly decreases to an acceptable level, namely, $1.4 \%$ or $0.9 \%$. This implies that a mesh with the characteristic resolution of $4 \mu \mathrm{m}$ is sufficient to capture the instability of oscillating combustion and is used in later simulations in this study.

By decreasing the sphere diameter to $D=10 \mathrm{~mm}$, another case that was thought to be free of oscillation, or in a steady state, had been simulated in previous studies. ${ }^{10,11}$ In contrast with the steady state, the present simulation reveals an unsteady combustion mode, as shown in Fig. 5. In the early stage of one cycle, a new flame front appears in front of the main flame front, as shown in Fig. 5(a). The new combustion region then becomes large and merges with the original flame front, as shown in Figs. 5(b) and 5(c). The merged

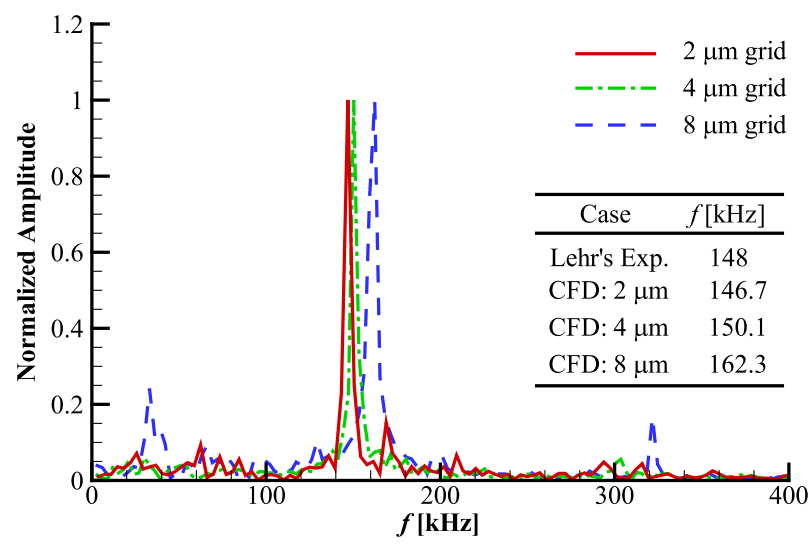

FIG. 4. Frequencies of oscillating combustion obtained with different grid resolutions in the case that $D=15 \mathrm{~mm}$. combustion zone expands downstream, while the flame front along the stagnation streamline recedes toward the stagnation point, until a new flame front of the next cycle appears, as shown in Figs. 5(d)5(f). This process is similar to that in the previous unstable combustion mode, ${ }^{10,11}$ and the partially merged flame front in Fig. 5(d) is similar to that in Fig. 2.

The newly observed unsteady combustion cannot be verified directly owing to a lack of experiments conducted for the same sphere diameters. To verify the unsteadiness arising in the case that $D=10 \mathrm{~mm}$, three other cases are simulated using $D=8 \mathrm{~mm}, 9 \mathrm{~mm}$, and $11 \mathrm{~mm}$. The temporal variations in the shock location presented in Fig. 6 clearly show that there is unsteady combustion in the cases that $D=9 \mathrm{~mm}, 10 \mathrm{~mm}$, and $11 \mathrm{~mm}$. The small diameter of $D=8 \mathrm{~mm}$ leads to a steady state, and weak oscillation is observed in the case that $D=9 \mathrm{~mm}$. In the case that $D=11 \mathrm{~mm}$, there is strong oscillation, as shown in Fig. 6. On the whole, both the averaged stand-off distance of the shock and the oscillation amplitude increase when the sphere diameter $D$ becomes large.

To get the features of unsteady combustion, the signals in Fig. 6 are processed adopting FFT, leading to the frequency distributions in Fig. 7. Note that to extract the oscillation frequency of unsteady shock-induced combustion more accurately, the signals of the initiation period are excluded in the FFT processes, and only the signals of the stably oscillating stage are analyzed. Generally speaking, there are two different oscillation patterns in the three cases that $D=9 \mathrm{~mm}, 10 \mathrm{~mm}$, and $11 \mathrm{~mm}$. In the case that $D=11 \mathrm{~mm}$, there is a dominant frequency of $171.4 \mathrm{kHz}$, which is slightly higher than that in the case that $D=15 \mathrm{~mm}$ (Fig. 4). In contrast, the dominant frequencies in the other two cases (plotted as red and blue curves) exceed $300 \mathrm{kHz}$ and are much higher than the dominant frequency in the case that $D=11 \mathrm{~mm}$. It had been found that ${ }^{14}$ there is a lower dominant frequency of around $30 \mathrm{kHz}$ when increasing the sphere diameter to approximately $D=20 \mathrm{~mm}$. The frequency of around $150 \mathrm{kHz}$ was therefore referred to as $\mathrm{HF}$, and a frequency of around $30 \mathrm{kHz}$ was referred to as LF in Ref. 14. The frequency of $\sim 300 \mathrm{kHz}$ is referred to as a super-high frequency (sHF) in the present study to maintain consistency with previous research studies. $^{9-11,14,15}$

To clarify the formations of HF and sHF unsteady combustion modes, the flow evolutions of the cases that $D=9 \mathrm{~mm}$ and $11 \mathrm{~mm}$ along the stagnation streamline are, respectively, illustrated in Figs. 8 and 9 , adopting $x-t$ diagrams. In these diagrams, the propagation of compression waves is clearly shown by contours of the pressure gradient, whereas the propagation of entropy waves, characterized by the continuous temperature gradient but not the pressure gradient, are easily identified from contours of the temperature gradient.

As for the formation mechanism of the HF unsteady combustion mode, the shock front and flame front are clearly characterized by periodical oscillations in Fig. 8. Accompanying oscillations of the shock front and flame front, compression waves and entropy waves are periodically generated and propagate between these fronts. Specifically, when a new flame front is generated upstream of an old flame front, a compression wave is generated concomitantly and propagates upstream. When the compression wave reaches and interacts with the shock front, the shock front moves upstream. Meanwhile, an entropy wave is reflected and propagates downstream. The reflected entropy wave has an obvious temperature peak (as shown by the red solid line in Fig. 10), which induces 

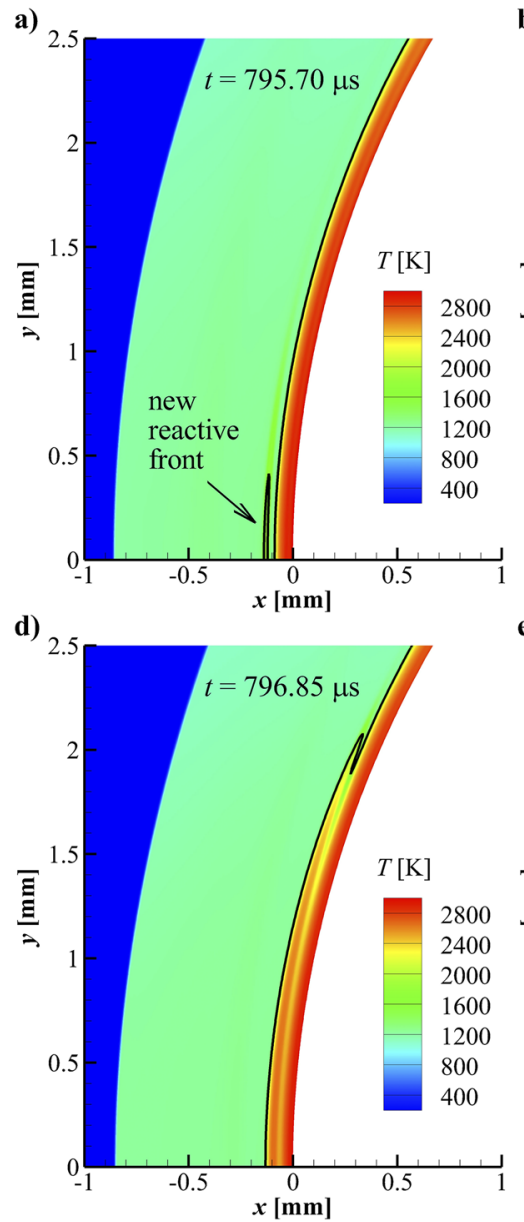
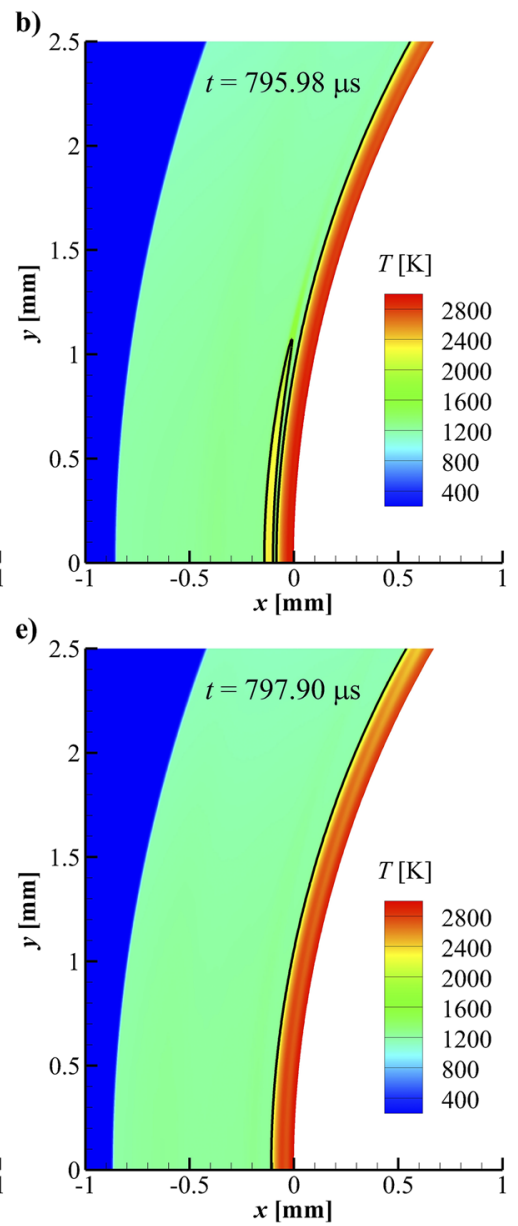
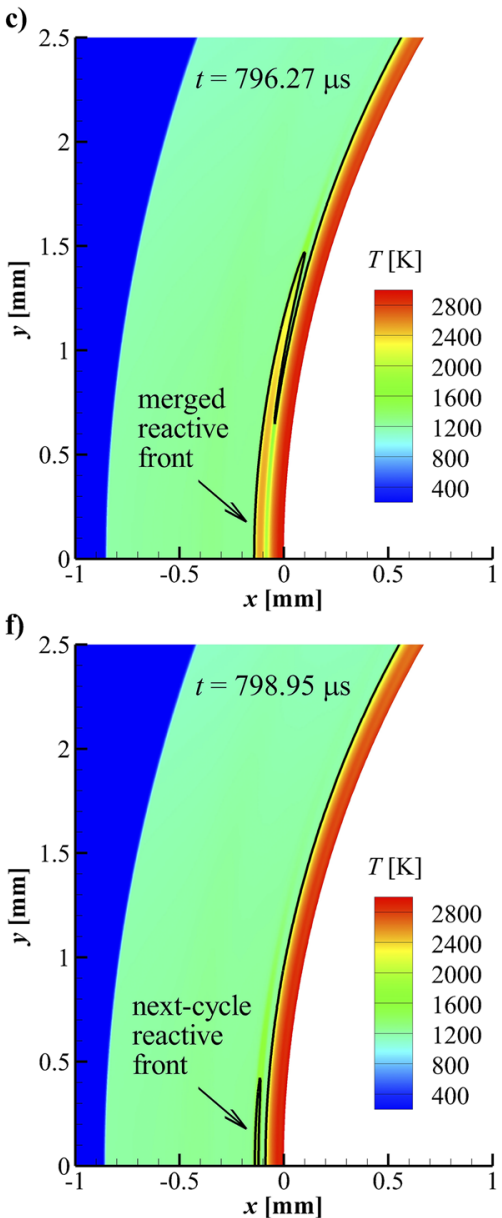

FIG. 5. Temperature contours (where black curves denote the $\mathrm{H}_{2}$ mass fraction isoline corresponding to half the inflow value) in the case that $D=10 \mathrm{~mm}$ at different time instants: (a) $t=795.70 \mu \mathrm{s}$, (b) $t=795.98 \mu \mathrm{s}$, (c) $t=796.27 \mu \mathrm{s}$, (d) $t=796.85 \mu \mathrm{s}$, (e) $t=797.90 \mu \mathrm{s}$, and (f) $t=798.95 \mu \mathrm{s}$.

another new flame front after propagating a certain distance downstream of the shock front. Figure 8 shows that new flame fronts form periodically, resulting in oscillation of the shock front. The wave dynamics discussed above are essentially the same as those observed

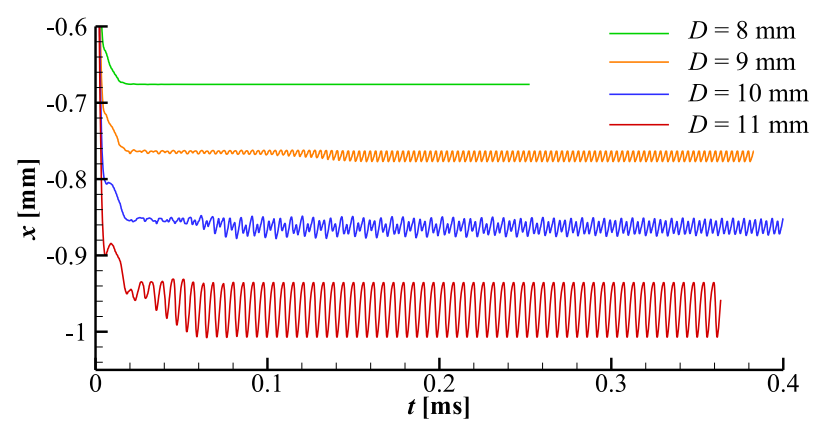

FIG. 6. Temporal variations of the shock location in the cases that $D=8 \mathrm{~mm}, 9 \mathrm{~mm}$, $10 \mathrm{~mm}$, and $11 \mathrm{~mm}$ by McVey and Toong ${ }^{25}$ and Matsuo et al., ${ }^{10,11}$ demonstrating a universal mechanism of the HF unsteady combustion mode.

As for the formation mechanism of the sHF unsteady combustion mode, quantitatively different wave dynamics are observed in

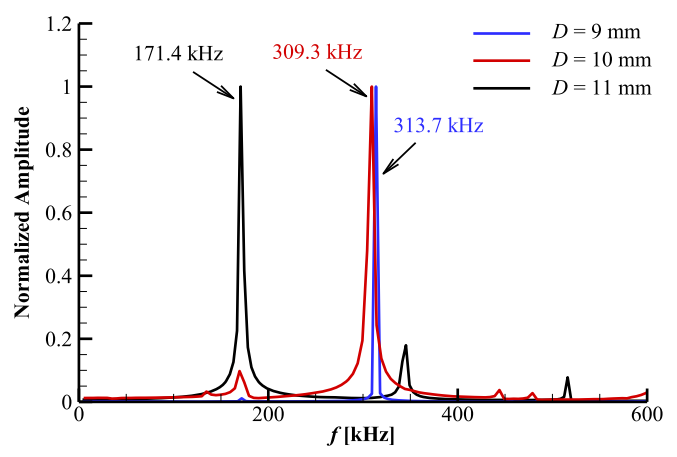

FIG. 7. Frequency distributions obtained by FFT of the shock location in the cases that $D=9 \mathrm{~mm}, 10 \mathrm{~mm}$, and $11 \mathrm{~mm}$. 
(a)

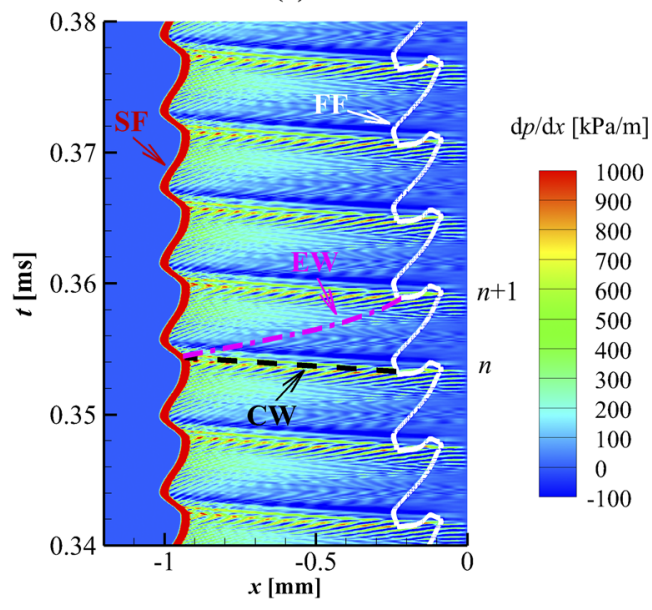

(b)

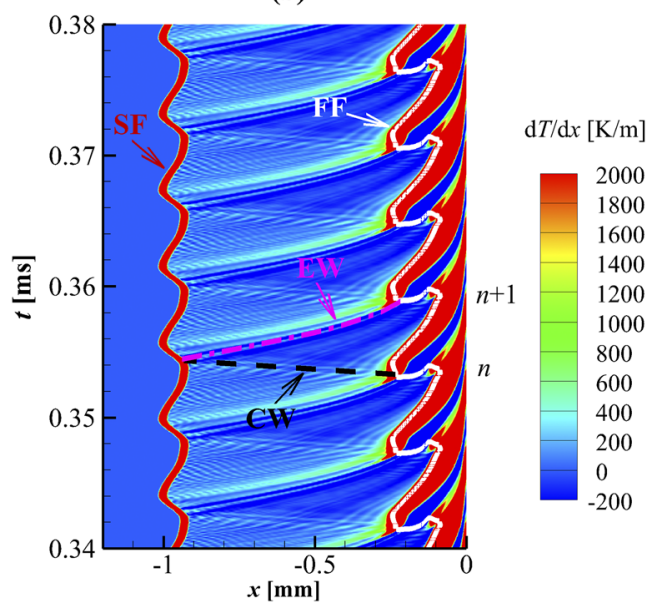

FIG. 8. The $x$ - $t$ diagrams of the pressure gradient (a) and temperature gradient (b) along the stagnation streamline in the case that $D=11 \mathrm{~mm}$ (SF: shock front; FF: flame front; CW: compression wave; and EW: entropy wave).

Fig. 9. In the case of the HF mode (Fig. 8), the entropy wave formed by the compression wave of the generation of the $n$th flame front generates the $(n+1)$ th flame front; in contrast, the entropy wave formed by the compression wave of the generation of the $n$th flame front generates the $(n+2)$ th flame front in the case of the sHF mode (Fig. 9). Correspondingly, the generation of the $(n+3)$ th flame front results from the compression wave of the $(n+1)$ th flame front in the case of the sHF mode. This staggered interaction feature of wave and flame front generation for the sHF mode changes the oscillation frequency to approximately twice that of the HF mode.

A transition from HF to sHF occurs when $D$ decreases, suggesting that the diameter affects the wave interaction. In the case of a small sphere diameter, such as $D=9 \mathrm{~mm}$, the flow in the stagnation zone is affected by a large expansion effect because of large curvature and flow strain. Hence, unsteady waves propagating between the shock and flame front weaken quickly, and the reflected entropy wave is not strong enough to form a new flame front directly. Thereafter, to induce the oscillation of the next cycle, the reflected entropy needs to be enhanced by other factors. In the case of the sHF mode, the factor is the compression wave of the next cycle. Figure 10 shows the temperature distribution along the stagnation line at $359.0 \mu \mathrm{s}$ for $D=9 \mathrm{~mm}$ (the blue dashed-dotted line). It is seen that there are two temperature peaks before the flame front, representing two entropy waves. The upstream wave is raised from the $(n+1)$ th cycle (a)

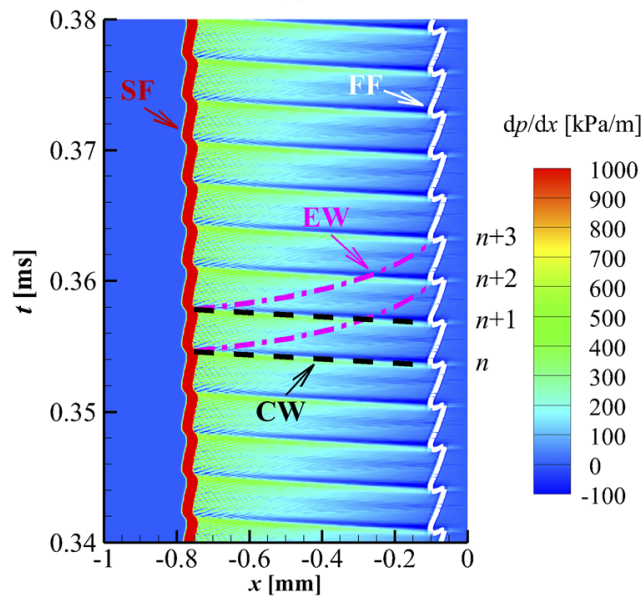

(b)

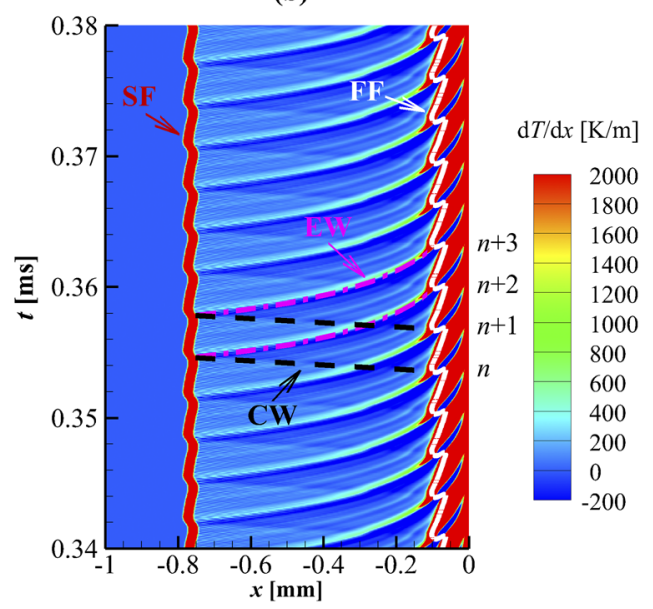

FIG. 9. The $x-t$ diagrams of the pressure gradient (a) and temperature gradient (b) along the stagnation streamline in the case that $D=9 \mathrm{~mm}$ (SF: shock front; FF: flame front; $\mathrm{CW}$ : compression wave; and EW: entropy wave). 


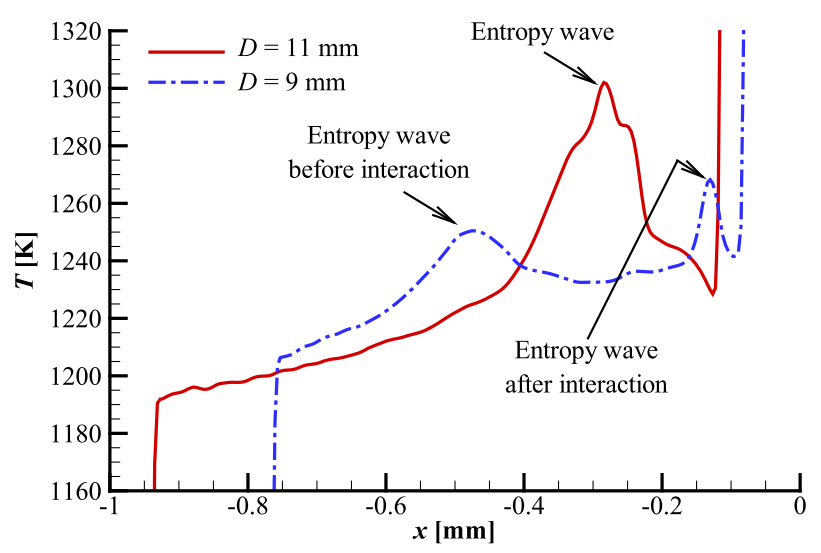

FIG. 10. Temperature along dashed lines in Fig. $8(t=358.2 \mu \mathrm{s}$ in the case that $D=11 \mathrm{~mm})$ and Fig. $9(t=359.0 \mu \mathrm{s}$ in the case that $D=9 \mathrm{~mm})$.

and has not interacted with the compression wave of the $(n+2)$ th cycle, while the downstream wave is raised from the $n$th cycle and has interacted with the compression wave of the $(n+1)$ th cycle. After enhancement by the compression wave, the temperature peak of the downstream entropy wave is larger than that of the upstream wave and is high enough to induce the formation of the new flame front of the $(n+2)$ th cycle finally. That is to say, the staggered interaction feature of waves and flame fronts is necessary for oscillation combustion in the case of a small sphere.

In the case that $D=11 \mathrm{~mm}$, the flow expansion effect in the stagnation zone has been weakened enough, and waves propagating between the shock and flame front are strong enough to induce combustion before the old flame front. Therefore, the formation of oscillation combustion no longer depends on the interaction of the entropy wave with the next-cycle compression wave. As shown in Fig. 10, the temperature peak of the entropy wave for $D=11 \mathrm{~mm}$ is obviously larger than that for $D=9 \mathrm{~mm}$. Thus, the staggered interaction feature disappears, and the oscillation combustion mode changes to the HF mode. As the compression waves are stronger for $D=11 \mathrm{~mm}$ (HF mode), the resulting amplitude of the shock front is larger than that for $D=9 \mathrm{~mm}$ (sHF mode).

The above results and related analysis are based on flow fields that have reached an equilibrium state, although unsteady combustion arises owing to the instability of shock and heat release coupling. However, we observe that a certain nonequilibrium state may exist, leading to different oscillation features. As shown in Fig. 11(a), a two-peak frequency distribution appears in the initial stage (Stage 1) of the $D=10 \mathrm{~mm}$ case. The lower frequency is $169.9 \mathrm{kHz}$, which is close to the dominant frequency in the case that $D=11 \mathrm{~mm}$ (that is, $171.4 \mathrm{kHz}$ ). However, after long evolution of time, the lower frequency disappears and only the higher frequency remains (Stage 2), as shown in Figs. 11(b) and 11(c). The frequency distribution of the nonequilibrium stage implies the existence of an intermediate case, illustrating the shift of the dominant frequency depending on the diameter.

According to the results of the present simulations, the latest diameter-frequency diagram for oscillating combustion induced by the curved shock of a sphere is plotted in Fig. 12. It is seen that the dominant frequency of oscillation does not continuously change as the sphere diameter increases but abruptly changes twice, resulting in three oscillation combustion modes: the HF mode, LF mode, and newly observed sHF mode. The first abrupt change, from the sHF mode to the HF mode, occurs at approximately $D=10 \mathrm{~mm}$, while the second abrupt change, from the HF mode to the LF mode, occurs at approximately $D=20 \mathrm{~mm}$. Within the diameter range of each mode, the oscillation frequency decreases slightly as the sphere

(a) Stage 1
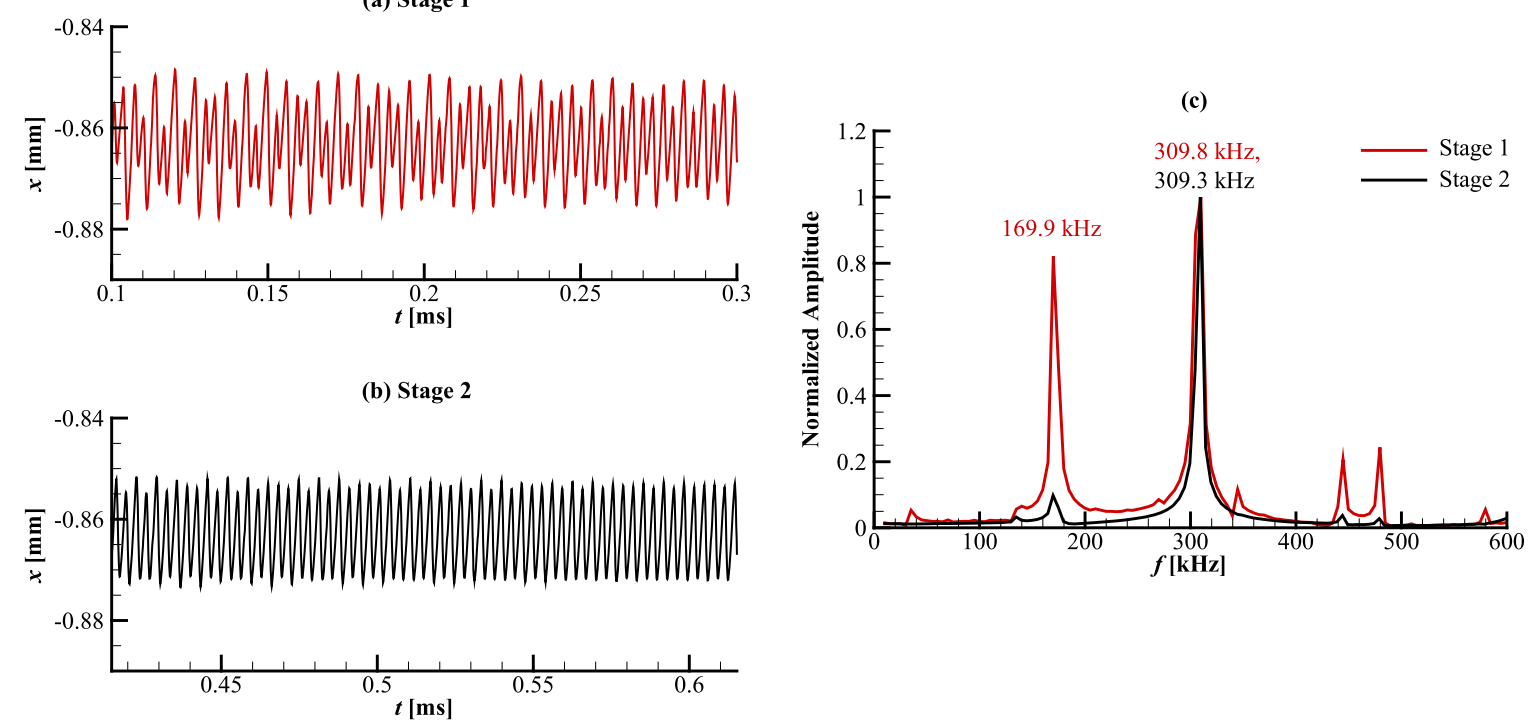

FIG. 11. Temporal variation of the shock location in the case that $D=10 \mathrm{~mm}$ : (a) stage 1; (b) stage 2; and (c) frequencies obtained through FFT of the shock location. 


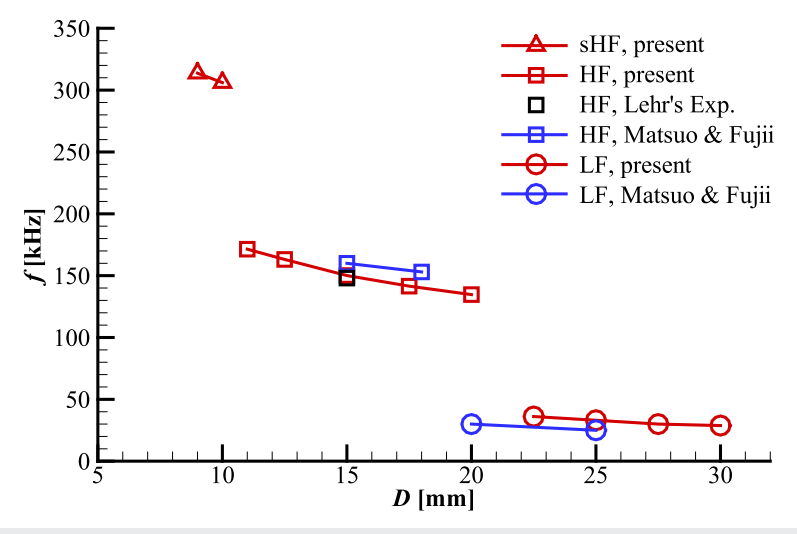

FIG. 12. Frequency of unsteady combustion depending on the sphere diameter.

size increases; this agrees with qualitative analysis of the problem as follows. As the sphere diameter increases, the expansion effect in the stagnation zone weakens and the temperature gradient along the stagnation line decreases, leading to a longer combustion induction time. Thereafter, the distance from the shock front to the flame front increases slightly with an increase in the sphere diameter. The above analysis reveals that the oscillation combustion is relative to the propagation of compression and entropy waves between these two fronts. A larger distance between the two fronts thus results in a longer propagation time of the compression and entropy waves for one cycle and, consequently, a lower oscillation frequency. For comparison, the experimental results of Lehr ${ }^{9}$ and previous simulation results of Matsuo and Fujii ${ }^{14}$ are presented in Fig. 12 as well. It is seen that the frequency levels of the HF and LF modes of the present simulations are basically the same as those obtained previously., ${ }^{9,14}$ One of the major differences is the unsteady combustion mode for $D=20 \mathrm{~mm}$. That is to say, the HF mode is observed in the equilibrium oscillation stage in the present simulation, whereas it was observed as the LF mode in the simulations of Matsuo and Fujii ${ }^{14}$ The main difference between the present study and the previous simulations ${ }^{10-15}$ is the newly observed sHF mode, whose mechanism relates to the staggered interaction feature of the wave interaction behind the shock front.

\section{CONCLUSION}

This paper reported a new unsteady combustion mode induced by the curved shock before a sphere in a high-speed hydrogen-air mixture. The dominant frequency of the new mode reaches approximately twice that of the original HF mode and is thus referred to as the sHF mode to remain consistent with terminology in previous works. The sHF mode appears in cases of a small sphere diameter, and with a proper diameter, there is a transition between the HF and sHF modes. According to pressure and temperature gradients along the stagnation streamline, the interaction of the shock and flame front was analyzed, revealing the mechanism of the sHF mode. For a small diameter, the weak compression wave affects the flame front of every other cycle through the reflected entropy wave, resulting in a staggered interaction feature of the waves in this mode.

\section{ACKNOWLEDGMENTS}

This research was supported by the National Natural Science Foundation of China (NSFC) (Grant No. 11822202).

\section{DATA AVAILABILITY}

The data that support the findings of this study are available within the article.

\section{REFERENCES}

${ }^{1}$ E. S. Oran, "Understanding explosions-From catastrophic accidents to creation of the universe," Proc. Combust. Inst. 35, 1-35 (2015).

${ }^{2}$ K. Xue, X. Shi, J. Zeng, B. Tian, P. Han, J. Li, L. Liu, B. Meng, X. Guo, and C. Bai, "Explosion-driven interfacial instabilities of granular media," Phys. Fluids 32, 084104 (2020).

${ }^{3}$ J. Urzay, "Supersonic combustion in air-breathing propulsion systems for hypersonic flight," Annu. Rev. Fluid Mech. 50, 593-627 (2018).

${ }^{4}$ C. Chen, T. Gao, and J. Liang, "Separation induced low-frequency unsteadiness in a supersonic combustor with single-side expansion," Phys. Fluids 31, 056103 (2019).

${ }^{5}$ V. Anand and E. Gutmark, "Rotating detonation combustors and their similarities to rocket instabilities,” Prog. Energy Combust. Sci. 73, 182-234 (2019).

${ }^{6}$ G. X. Xiang, X. Gao, W. J. Tang, X. Z. Jie, and X. Huang, "Numerical study on transition structures of oblique detonations with expansion wave from finitelength cowl," Phys. Fluids 32, 056108 (2020).

${ }^{7}$ G. Xiang, H. Li, R. Cao, and X. Chen, "Study of the features of oblique detonation induced by a finite wedge in hydrogen-air mixtures with varying equivalence ratios," Fuel 264, 116854 (2020).

${ }^{8}$ Y. Liu, W. Zhou, Y. Yang, Z. Liu, and J. Wang, "Numerical study on the instabilities in $\mathrm{H}_{2}$-air rotating detonation engines," Fluids 30, 046106 (2018).

${ }^{9}$ H. F. Lehr, "Experiments on shock induced combustion," Astronaut. Acta 17, 589-597 (1972).

${ }^{10}$ A. Matsuo and T. Fujiwara, "Numerical investigation of oscillatory instability in shock-induced combustion around a blunt body," AIAA J. 31, 1835-1841 (1993).

${ }^{11}$ A. Matsuo, K. Fujii, and T. Fujiwara, "Flow features of shock-induced combustion around projectile traveling at hypervelocities," AIAA J. 33, 1056-1063 (1995).

${ }^{12}$ A. Matsuo and K. Fujii, "Detailed mechanism of the unsteady combustion around hypersonic projectiles,” AIAA J. 34, 2082-2089 (1996).

${ }^{13}$ J.-Y. Choi, I.-S. Jeung, and S. Lee, "Dimensional analysis of the effect of flow conditions on shock-induced combustion," Symp. (Int.) Combust. 26, 2925-2932 (1996).

${ }^{14}$ A. Matsuo and K. Fujii, "Prediction method of unsteady combustion around hypersonic projectile in stoichiometric hydrogen-air," AIAA J. 36, 1834-1841 (1998).

${ }^{15}$ J.-Y. Choi, I.-S. Jeung, and Y. Yoon, "Computational fluid dynamics algorithms for unsteady shock-induced combustion. I-Validation,” AIAA J. 38, 1179-1187 (2000).

${ }^{16}$ Y. Uda and A. Matsuo, "Three-dimensional numerical simulation of shockinduced combustion around a blunt body," in 21st International Colloquium on the Dynamics of Explosions and Reactive Systems (Poitiers, France, 2007).

${ }^{17}$ B. J. McBride, S. Gordon, and M. A. Reno, "Coefficients for calculating thermodynamic and transport properties of individual species," Technical Report No. NASA TM-4513, 1993.

${ }^{18} \mathrm{C}$. J. Jachimowski, "An analytical study of the hydrogen-air reaction mechanism with application to scramjet combustion," Technical Report No. NASA TP-2791, 1988.

${ }^{19}$ J.-Y. Choi, E. J.-R. Shin, and I.-S. Jeung, "Unstable combustion induced by oblique shock waves at the non-attaching condition of the oblique detonation wave," Proc. Combust. Inst. 32, 2387-2396 (2009). 
${ }^{20}$ Z. Zhang, K. Ma, W. Zhang, X. Han, Y. Liu, and Z. Jiang, "Numerical investigation of a Mach 9 oblique detonation engine with fuel pre-injection," Aerosp. Sci. Technol. 105, 106054 (2020).

${ }^{21}$ C. Fureby, M. Chapuis, E. Fedina, and S. Karl, "CFD analysis of the HyShot II scramjet combustor," Proc. Combust. Inst. 33, 2399-2405 (2011).

${ }^{22} \mathrm{~S}$. Chakravarthy, "A unified-grid finite volume formulation for computational fluid dynamics," Int. J. Numer. Methods Fluids 31, 309-323 (1999).
${ }^{23}$ E. F. Toro, Riemann Solvers and Numerical Methods for Fluid Dynamics: A Practical Introduction (Springer Science \& Business Media, 2013).

${ }^{24}$ D. L. Ropp and J. N. Shadid, "Stability of operator splitting methods for systems with indefinite operators: Advection-diffusion-reaction systems," J. Comput. Phys. 228, 3508-3516 (2009).

${ }^{25} \mathrm{~J}$. B. McVey and T. Y. Toong, "Mechanism of instabilities of exothermic hypersonic blunt-body flows," Combust. Sci. Technol. 3, 63-76 (1971). 